\title{
ATENDIMENTO À PESSOA SURDA QUE UTILIZA A LÍNGUA DE SINAIS, NA PERSPECTIVA DO PROFISSIONAL DA SAÚDE
}

Neuma Chaveiro' , Maria Alves Barbosa ${ }^{2}$, Celmo Celeno Porto ${ }^{3}$, Denise Bouttelet Munari ${ }^{4}$, Marcelo Medeiros ${ }^{5}$, Soraya Bianca Reis Duarte ${ }^{6}$

RESUMO: Pesquisa descritivo-analítica, com abordagem qualitativa, que teve como objetivo caracterizar a comunicação dos profissionais da saúde com a pessoa surda e descrever recursos de relacionamento. O corpus foi composto por sete profissionais da saúde, os dados foram coletados no Município de Goiânia, analisados pela técnica de análise de conteúdo e identificadas duas categorias: Comunicação entre profissionais da saúde e pessoas surdas que utilizam a língua de sinais, e Recursos de relacionamento com a pessoa surda. Na primeira categoria, verificou-se a Língua Brasileira de Sinais (LIBRAS) como relevante instrumento de comunicação no atendimento à pessoa surda. Na segunda categoria, destaca-se a atuação do Tradutor intérprete de LIBRAS. Constatou-se que os profissionais não estão suficientemente preparados para cuidar da pessoa surda e conclui-se que a comunicação verbal não tem sido suficiente para estabelecer o vínculo entre esses.

PALAVRAS-CHAVE: Surdez; Comunicação; Linguagem de sinais.

\section{CARING FOR A PERSON THAT USES DEAF SIGN LANGUAGE IN THE PERSPECTIVE OF THE HEALTH PROFESSIONAL}

\begin{abstract}
This is a descriptive and analytical research with a qualitative approach, which aims to characterize the communication of health professionals with the deaf person and describe features of the relationship. The corpus was composed of seven professionals, data were collected in Goiânia, and they were analyzed using content analysis. Two categories were analyzed: communication between healthcare professionals and deaf people using sign language, and relationship resources with the deaf person. In the first category, the Brazilian Sign Language (LIBRAS) was pointed out as an important communication tool in caring of deaf people. In the second category, the work of the translator and interpreter of LIBRAS was pointed out. It was found that professionals are not adequately prepared to care for the deaf person and it is concluded that verbal communication has not been sufficient to establish the link between them. KEYWORDS: Deafness; Communication; Sign language.
\end{abstract}

\section{ATENDIMIENTO A LA PERSONA SORDA QUE UTILIZA LA LENGUA DE SEÑALES, EN LA PERSPECTIVA DEL PROFESIONAL DE LA SALUD}

RESUMEN: Investigación descriptivo-analítica con abordaje cualitativa cuyo objetivo fue caracterizar la comunicación de los profesionales de la salud con la persona sorda, así como describir recursos de relaciones. El corpus fue composto por siete profesionales de la salud, y los datos fueron recogidos en el municipio de Goiânia, analizados por la técnica de análisis de contenido e identificadas dos categorías: Comunicación entre profesionales de la salud y personas sordas que utilizam a língua de sinais, y Recursos de relacionamento con a persona sorda. En la primera categoría, se ha verificado que la Lengua Brasileña de Siñales (LIBRAS) es relevante como instrunento de comunicación en atendimiento a la persona sorda. En la segunda categoría, se destaca la actuación del Traductor intérprete de LIBRAS. Se constató que los profesionales no están listos de modo suficiente para el cuidar de la persona sorda y se concluye que la comunicación verbal no hay sido suficiente para establecer el vínculo entre estes.

PALABRAS CLAVE: Sordez; Comunicación; Lenguaje de signos.

${ }^{1}$ Fonoaudióloga. Doutoranda em Ciências da Saúde. Professora da Faculdade de Letras/LIBRAS da Universidade Federal de Goiás-UFG. ${ }^{2}$ Enfermeira. Doutor em Enfermagem. Professor Adjunto da Faculdade de Enfermagem da UFG.

${ }^{3}$ Médico. Professor Emérito da Faculdade de Medicina da UFG.

${ }^{4}$ Enfermeira. Doutor em Enfermagem. Professor Titular da Enfermagem da UFG.

${ }^{5}$ Enfermeiro. Doutor em Enfermagem. Professor Associado da Faculdade de Enfermagem da UFG.

${ }^{6}$ Fonoaudióloga. Mestranda do Programa de Pós-Graduação em Ciência da Saúde - UFG. Coordenador do Curso de Graduação Letras/LIBRAS da Universidade Federal de Santa Catarina.

Autor correspondente:

Neuma Chaveiro

Universidade Federal de Goiás

Av. K,138 - 74075200 - Goiânia-GO-Brasil

Recebido: $17 / 01 / 10$

E-mail: neumachaveiro@hotmail.com

Aprovado: 23/07/10 


\section{INTRODUÇÃO}

Dados do Instituto Brasileiro de Geografia e Estatística (IBGE) de 2000 revelam que existem, no Brasil, 24,5 milhões de pessoas com deficiência, correspondendo a $14,5 \%$ da população. Destas, $16,7 \%$ apresentam deficiência auditiva, ou seja, são 5.735.099 de pessoas surdas ${ }^{(1)}$.

A comunidade surda é composta por pessoas que usam Língua de Sinais (LS) como primeiro meio de comunicação, tendo cultura própria e características únicas; a LS consiste em linguagem além de normas sociais $^{(2)}$.

As LS, embora presentes em todos os continentes, não têm uma estrutura universal: apresentam estrutura gramatical diferenciada. É uma linguagem de modalidade espaço-visual, pois o sistema de signos compartilhado é recebido pelos olhos e sua produção é realizada pelas mãos, no espaço. São reconhecidas como língua pela Linguística e possuem uma estruturação tão complexa como as línguas faladas, fato que lhes permite ser atribuido o conceito de línguas naturais $^{(3)}$.

A Língua Brasileira de Sinais (LIBRAS) teve sua origem na Língua de Sinais Francesa ${ }^{(2)}$, e foi reconhecida como meio legal de comunicação e expressão da comunidade surda pela Lei Federal $n$. $10.436 / 2002$, a qual foi regulamentada pelo Decreto n. 5.626 , de 22 de dezembro de $2005^{(4)}$.

A partir do momento em que os países reconhecem oficialmente a LS como língua natural da comunidade surda, o profissional da saúde passa a ser solicitado a se preparar para dar efetivo atendimento a esta população ${ }^{(2,5)}$.

$\mathrm{Na}$ área de saúde, habilidades de comunicação interpessoal são imprescindíveis na assistência a qualquer paciente, e as ações dos profissionais da saúde são pautadas pela comunicação, independente da sua formação acadêmica. Este profissional tem como ferramenta-base de seu trabalho as relações humanas ${ }^{(6)}$. Portanto, compreender o relacionamento entre o profissional da saúde e a pessoa surda é condição necessária para qualificar os serviços prestados à população surda.

$\mathrm{O}$ encontro clínico entre o profissional da saúde e a pessoa surda normalmente acontece fora dos padrões esperados na rotina de qualquer profissional; indivíduos surdos e profissionais se vêem diante de limitações que dificultam o vínculo a ser estabelecido entre eles. Esse aspecto se agrava se for considerado o fato de que a LS quase sempre é desconhecida pelos profissionais de saúde. Para muitos, a solução encontrada está na solicitação da presença do intérprete da LS como mediador, o que implica na inclusão de uma terceira pessoa nesta relação ${ }^{(7-8)}$.

Por outro lado, pouco se discute sobre a importância de conhecimentos mínimos dos profissionais de saúde sobre a LS, para que esses possam qualificar seu contato com a pessoa surda como mais uma forma de inclusão $o^{(9)}$. Assim, viabilizar a acessibilidade é uma questão fundamental que os profissionais da saúde devem se atentar, como forma de inclusão das pessoas com deficiências, de modo a garantir uma ação terapêutica de qualidade, tanto quanto como aquela realizada com pessoas consideradas normais ${ }^{(10)}$.

Diante da complexidade que envolve a relação entre a pessoa surda e o profissional da saúde, perguntamos: sendo a comunicação, ferramenta essencial na execução dos mais variados procedimentos na área da saúde, como os profissionais e pessoas surdas interagem? Como se caracteriza essa comunicação?

O objetivo deste estudo foi caracterizar a comunicação de profissionais da saúde com a pessoa surda que utiliza a LS e descrever os recursos de relacionamento utilizados pelos profissionais da saúde no atendimento.

\section{METODOLOGIA}

Este estudo faz parte de um projeto de pesquisa cujo objetivo geral é investigar o atendimento à pessoa surda na área da saúde. O projeto contém três pesquisas e foi submetido ao Comitê de Ética em Pesquisa Médica Humana e Animal do Hospital das Clínicas da Universidade Federal de Goiás, e aprovado no ano de 2005, sob n. 126/05. A primeira pesquisa foi realizada no ano de 2006 e analisou o atendimento do paciente surdo na visão da pessoa surda. O segundo estudo, feito no ano de 2007, investigou a percepção do intérprete da LS na assistência à pessoa surda. A terceira pesquisa, concluída no ano de 2009 e descrita neste artigo, analisa o atendimento à pessoa surda sob a perspectiva do profissional da saúde.

Trata-se de uma pesquisa de natureza descritivo-analítica com abordagem qualitativa, apropriada para este estudo por privilegiar significados, experiências, sentimentos, atitudes e valores dos sujeitos envolvidos, num espaço mais profundo das relações, considerando como objeto de estudo pessoas pertencentes a determinada condição social, com suas crenças e valores ${ }^{(11)}$. 
A pesquisa foi desenvolvida numa Instituição que atende pessoas com deficiência auditiva, visual, motora e mental, situada no município de Goiânia, Estado de Goiás, Brasil. O trabalho de reabilitação nessa instituição é realizado por uma equipe multiprofissional.

A população deste estudo foi constituída por sete profissionais da saúde, sendo dois fonoaudiólogos, dois fisioterapeutas, dois psicólogos e um dentista, com idades variando entre 33 e 55 anos. Foram definidos como critérios de inclusão: ter idade acima de 18 anos, ser profissional da saúde e trabalhar na instituição do estudo, atender pessoas surdas e se dispor a participar da pesquisa assinando o Termo de Consentimento Livre e Esclarecido, conforme o que determina a Resolução n. 196/96.

De acordo com o método adotado, o número dos sujeitos da pesquisa não foi pré-determinado, mas definido a partir da observação da ocorrência de repetição dos conteúdos, ou seja, da saturação dos dados, que então foram considerados suficientes para compreensão dos fenômenos estudados ${ }^{(11)}$.

A coleta de dados ocorreu entre agosto e novembro de 2008, por meio de entrevista semiestruturada, e o instrumento continha questões relacionadas à comunicação e à descrição da relação estabelecida entre estes profissionais e a pessoa surda. As entrevistas, com duração média de 20 minutos cada, foram gravadas e transcritas pelos autores.

A análise dos dados foi fundamentada na abordagem qualitativa e sistematizada por meio de uma aproximação à técnica de Análise de Conteúdo Categorial Temática, proposta por Bardin, que "consiste em descobrir os 'núcleos de sentido' que compõem a comunicação e cuja presença ou frequência de aparição pode significar alguma coisa para o objetivo analítico escolhido"(12:105).

Para identificar os relatos dos sujeitos entrevistados, e garantir o anonimato quanto à identidade, foi utilizada a letra inicial da palavra sujeito (S), seguida do número de acontecimento das entrevistas (S1, S2, S3 etc.).

\section{RESULTADOS E DISCUSSÃO}

O tratamento dos dados permitiu identificar duas categorias temáticas: Comunicação entre profissionais da saúde e pessoas surdas que utilizam a Língua de Sinais e Recursos de relacionamento com a pessoa surda.

\section{Comunicação entre profissionais da saúde e pessoa surda que utiliza a Língua de Sinais}

Essa categoria revela que a comunicação é uma ferramenta essencial nos mais variados procedimentos da área de saúde. Ao atender uma pessoa surda, os profissionais da saúde se deparam com dificuldades para estabelecer uma comunicação eficaz. O fato de não dominar a LS constitui uma barreira para a interação com a pessoa surda.

Os sujeitos da pesquisa tentam utilizar a LS no encontro clínico, mas a fluência é limitada, sendo que, dos entrevistados, cinco não dominavam a LS, mas reconheciam que esta possibilita o desenvolvimento linguístico, social e intelectual das pessoas surdas, bem como a integração no grupo social ao qual pertencem.

A LS é um instrumento de comunicação de grande importância no atendimento à pessoa surda, porém, quase sempre não é compreendida por aqueles que prestam assistência na área de saúde ${ }^{(8-9)}$. Mesmo com o programa de capacitação permanente ao aprendizado da LIBRAS, como o da Instituição onde a pesquisa foi realizada, os profissionais apresentam dificuldades, pois a fluência na LS exige dedicação, como no aprendizado das Línguas Orais. Esse aspecto foi evidenciado nas falas de todos os sujeitos da pesquisa, destacando-se:

A comunicação fica muito restrita, vai depender só do que o paciente consegue oralizar, ler nos lábios e alguns gestos muito rudimentares. Seria muito interessante que os profissionais da saúde tivessem maior conhecimento da LS, dessa forma teria maior integração, teria a inclusão que todo mundo busca. (S1)

Na área da saúde, quando a pessoa não tem a noção da LS, fica difícil a comunicação. Ai, tem que usar gestos naturais e dificulta muito a compreensão [...]. (S7)

A LS é um dos elementos marcantes da cultura dos surdos, transmite crenças e valores específicos, sendo uma das principais características da identidade de um povo surdo. É uma forma de comunicação que capta as experiências visuais das pessoas surdas, proporcionando a comunicação interpessoal ${ }^{(13)}$ :

Antes quando não sabia a $L S$, tentava comunicar com alguns gestos universais, mas era muito complicado, 
muito difícil, porque às vezes a gente tem intenção de fazer alguma coisa, pede, mas ele não compreende [...]. (S2)

Eu uso LIBRAS, mas como sei pouco, foi um trabalho que tive muita dificuldade; trabalhei com criança surda. Eu tentava que ela me entendesse, mas foi muito difícil [...]. (S3)

Fica evidente nos depoimentos dos sujeitos da pesquisa que a LS é um recurso de comunicação que precisa ser conhecido e valorizado na prática das ações em saúde. Mesmo que não se conheça a LS, é fundamental interpretar seus aspectos suprassegmentais, que incluem gestos, expressões faciais e corporais ${ }^{(7-9)}$.

Problemas de comunicação interpessoal estão presentes em todo sistema de saúde e tornam-se mais significantes quando englobam barreiras de linguagem e cultura, conforme descrito no relato:

Não conseguiram compreender tudo o que eu queria passar, e eu não consegui compreender tudo que eles queriam me passar, porque não domino a LS, e muitas vezes eles chegam até nós sem nem uma forma de comunicação [...]. (S1)

O depoimento acima revela que a comunicação estabelecida entre profissionais da saúde e pessoas surdas não está propiciando a interação, podendo prejudicar a abordagem terapêutica e, consequentemente, o tratamento dispensado ao paciente.

Comunicar as questões relacionadas ao diagnóstico e ao tratamento é um dever dos profissionais da saúde e um direito dos pacientes ${ }^{(14)}$. Entretanto, diante de pessoas surdas que utilizam a LS, essa questão está comprometida pela dificuldade de comunicação, como assim se expressaram estas pessoas:

O profissional pode dar uma orientação, um diagnóstico, contrário ao que o paciente vem queixando, por falta de compreensão da linguagem. (S2)

É preciso estudar o paciente, mas também quem é a pessoa surda. Como ela faz a vinculação, como ela expressa sua frustração [...]. A comunicação não é uma comunicação efetiva, como acontece com pacientes ouvintes. (S5)

Eu sinto dificuldade. Primeiro, porque a gente atende de máscara. A gente tem de comunicar, e muitas vezes não temos o curso de Libras, que seria fundamental fazer isso. (S6)

A concepção socioantropológica da surdez pressupõe considerá-la não como uma deficiência a ser curada, eliminada ou normalizada, e sim como uma diferença a ser respeitada, e a pessoa surda como pertencente a uma comunidade minoritária que partilha uma LS, valores culturais, hábitos e modos de socialização próprios ${ }^{(3,15)}$. Para os profissionais que trabalham diretamente com pessoas surdas, como os da instituição deste estudo, informações sobre a cultura das pessoas surdas são primordiais:

Nós, profissionais da saúde, em especial os que trabalham com os surdos, devemos conhecer mais a pessoa surda [...]. (S5)

Eu vejo o surdo muito sozinho, isolado no meio social. Não percebo uma inclusão social acontecendo, por causa da dificuldade de comunicação. Se tivesse conhecimento da linguagem, uma base anterior antes de começar a atender [...]. (S3)

A especialização crescente dos profissionais da saúde resulta na fragmentação do cuidado de saúde, fazendo com que o paciente receba assistência de vários profissionais sem formar vínculo com nenhum deles. Afinal o ser humano não é a soma de suas partes. Pelo contrário, é uma unidade da qual o ser biológico, psíquico e social não podem ser separados ${ }^{(14)}$. Um profissional da saúde assim abordou a questão:

Como a pessoa é surda, a gente acha que só não tem a audição e que tudo funciona normalmente, a maneira dele enxergar o mundo, vivenciar, ter com experiências o mundo é diferente. Isso é real [...]. (S5)

É necessário melhorar a comunicação entre profissionais da saúde e pessoas surdas. A comunicação inadequada se torna uma barreira, portanto, é indispensável encontrar formas de interação para garantir uma assistência de melhor qualidade.

\section{Recursos de relacionamento com a pessoa surda}

Essa categoria revela que a presença do intérprete de LS em alguns momentos é valorizada, enquanto, e em outros, parece dificultar, apresenta também a necessidade de formação dos profissionais 
da saúde para atender a população surda que utiliza LS para se comunicar.

Imagina-se que a presença do intérprete de LS nos serviços de saúde solucionaria todos os problemas de comunicação entre a pessoa surda e o profissional de saúde. Entretanto, verifica-se que nem sempre é assim que ocorre. A atuação do intérprete pode melhorar, mas não é decisiva para um atendimento de qualidade $^{(7-9)}$, como colocou um sujeito da pesquisa:

O paciente pode se sentir constrangido com mais outra pessoa na sala. O ideal seria que não houvesse necessidade do intérprete, mas é melhor ter o intérprete e resolver a situação do que não ter. (S5)

Nesse relato, verifica-se que, ao traduzir as informações do profissional da saúde à pessoa surda, a responsabilidade do intérprete torna-se maior, devido às questões éticas que exigem as diversas situações na área da saúde. Portanto, o intérprete, além da função de traduzir fielmente a comunicação entre os sujeitos envolvidos, deve respeitar as questões confidenciais de cada pessoa. Para exemplificar melhor esta questão, destaca-se a seguinte manifestação de um profissional da saúde:

Seria ideal que não tivesse a presença do intérprete. Que eu como psicóloga soubesse LIBRAS, e fizesse dela um uso, que fosse entendivel para o paciente, porque tem a questão do sigilo, a questão vincular [...] seria bem mais tranquilo. Mas, numa situação de emergência, se o intérprete tiver sigilo, for realmente profissional, passar as coisas como devem ser passadas, não vejo problema, mas o intérprete precisa saber que ele é apenas intérprete. (S5)

Algumas pessoas surdas são relutantes quanto ao uso do intérprete, especialmente se ele conhece ou participa da comunidade surda na qual está inserido $^{(16)}$. Os surdos sentem-se ansiosos em relação a questões confidenciais, mas, sem um intérprete, ficam sem informações sobre decisões e condução da assistência a ser oferecida.

Outro entrevistado assim se posicionou sobre a presença do intérprete de LS:

Não utilizei o intérprete dentro do atendimento, mas para interpretar alguns testes psicológicos eu precisei do apoio do intérprete, para saber como me comunicaria com o paciente. Dentro da sessão não utilizei, mas penso que se tiver o consentimento do paciente, seria positiva a presença do intérprete, embora seja um momento muito intimo, e nem sempre é possivel a presença de outra pessoa. (S3)

Ao analisar a presença de um intérprete de LS, verifica-se a complexidade que envolve esta ação, pois não é suficiente ser conhecedor da LS, mas é necessário, também, respeitar o sigilo, saber transmitir o conteúdo da mensagem ${ }^{(17)}$. $\mathrm{Na}$ área de saúde existem muitos termos relacionados às especialidades e a linguagem técnica, muitas vezes, não é acessível aos intérpretes que não lidam com questões de saúde. Tal fato fica claro na observação de um profissional:

Ser vista como uma pessoa incompleta, uma pessoa que não sabe das coisas, e se o intérprete não conhecer realmente a pessoa surda, pensa que ele é completude do que falta no surdo. Falta um conhecimento real de quem é o surdo. (S5)

Os intérpretes da LS não têm ainda uma formação adequada para atuar na área de saúde. Espera-se que os cursos oferecidos em graduação e pós-graduação em todo o Brasil possam reverter este quadro, capacitando-os para atuar de modo mais profissional. Assim, as pessoas surdas poderão usufruir, sem constrangimento ou receio, da presença do profissional intérprete da $\operatorname{LS}^{(7-17)}$.

Os profissionais que trabalham na instituição onde a pesquisa foi realizada geralmente possuem formação para atuar na assistência a pessoas com deficiências. Porém, o conhecimento da LIBRAS não é critério para admissão do profissional, pois seu ensino nas Instituições de Ensino Superior somente é obrigatório nos cursos de licenciatura, sendo optativo nos demais ${ }^{(4)}$.

Os sujeitos da pesquisa argumentam que os profissionais da saúde não estão suficientemente preparados para cuidar da pessoa surda; justificam que na formação acadêmica o currículo não contempla as habilidades necessárias para atender essa população. Tal afirmação fica evidente no depoimento de um profissional, que assim se expressou:

Para favorecer a relação deve-se iniciar na formação acadêmica, ser mais divulgada a LS. Todo profissional pode um dia receber um paciente surdo e precisa estar apto para atendê-lo. (S3) 
Para o profissional de saúde, uma efetiva comunicação propicia um atendimento de melhor qualidade $^{(7-8)}$, portanto a capacitação desses profissionais para atender as pessoas surdas é uma necessidade urgente. Nesse sentido, espera-se que essa formação contemple a cultura dos surdos, noções básicas de LS e como se posicionar frente ao atendimento da pessoa surda. Esses aspectos podem assegurar o acesso aos cuidados de saúde, como assim explicitaram os sujeitos da pesquisa:

Precisava ter no currículo, não só sobre a LS, mas o conhecimento das pessoas com deficiências, as limitações. (S3)

O curso de LIBRAS seria muito importante para poder falar uma linguagem semelhante à dele. É fundamental, um fator que poderia ajudar bastante. O preparo dos profissionais para atender esse tipo de clientela, é uma clientela totalmente diferenciada, não tem como dialogar, usar a fala. (S6)

Fica claro nos depoimentos que existe uma lacuna na formação dos profissionais da saúde para atender as pessoas surdas. A maioria dos cursos na área de saúde caracteriza a surdez apenas como condição patológica, não compreendendo a população surda como um grupo minoritário e que tem uma cultura própria ${ }^{(7,17-18)}$.

As atividades de um profissional da saúde requerem conhecimentos bem mais do que apenas competência técnica, além das atribuições específicas, para buscar estabelecer com os pacientes relações de caráter interpessoal ${ }^{(6)}$.

Os profissionais da saúde, sujeitos da pesquisa, ressaltam que utilizam todos seus conhecimentos para estabelecer uma relação eficaz, como se pode observar nos depoimentos a seguir:

\section{Utilizei todos os recursos possiveis para me comunicar [...]. (S4)}

Se você propicia o trabalho do surdo a partir do significado, fica muito bom para quem está trabalhando com o surdo e para o próprio surdo também. (S7)

A aliança terapêutica, sempre necessária ao êxito do tratamento, está vinculada à compreensão do que o outro quer expressar ${ }^{(6)}$. Neste sentido a formação do profissional da saúde para atender a pessoa surda que utiliza a LS é imprescindível.

\section{CONCLUSÕES}

$\mathrm{O}$ atendimento à pessoa surda é um desafio para os profissionais da saúde e para o próprio surdo. O uso da linguagem verbal precisa ser substituído por outro recurso de comunicação, a LS. Sendo a LIBRAS a língua pela qual o surdo se expressa, os profissionais da saúde, especialmente os que trabalham em centros especializados na assistência à pessoa surda, como os da Instituição deste estudo, necessitam estudá-la ou pelo menos adquirir noções básicas da LS. Ademais, os profissionais da saúde precisam conhecer as particularidades culturais e linguísticas da comunidade surda, sem o que não conseguirá desenvolver habilidades comunicativas que favoreceram as relações interpessoais.

É necessário que as Instituições acadêmicas oportunizem aos futuros profissionais o aprendizado da LS, atendendo as necessidades da pessoa surda. A comunicação verbal utilizada pelos profissionais da saúde não é suficiente para estabelecer o vínculo, sendo, portanto, ineficaz, e podendo levar a erros no diagnóstico das doenças e no tratamento.

A construção de uma prática em saúde que transforme a assistência à pessoa surda irá acontecer por meio de estudos, ações e vontade política; assim, será possível reorganizar o atendimento, segundo princípios que têm como alicerce o intercâmbio das diferenças individuais e o convívio com a pluralidade humana.

\section{REFERÊNCIAS}

1. Instituto Brasileiro de Geografia e Estatística (IBGE). Censo Demográfico Brasileiro 2000. [acesso em 10 out 2008]. Disponível: http://www.ibge.com.br/.

2. Federação Nacional de Educação e Integração dos Surdos (FENEIS). LIBRAS: Língua Brasileira de Sinais. [acesso em 15 nov 2008]. Disponível: http:// www.feneis.br/

3. Quadros RM, Kanopp LB. Língua de sinais brasileira. Estudos linguísticos. Porto Alegre: Artmed; 2004.

4. Brasil. Decreto n. 5.626, de 22 de dezembro de 2005: regulamenta a Lei n. 10.436, sancionada em 24 de abril de 2002, a qual dispõe sobre a Língua Brasileira de Sinais - Libras e o art. 18 da Lei n. 10.098, de 19 de dezembro de 2000. Diário Oficial da República Federativa do Brasil, Brasília, 23 dez. 2005.

5. Lezzoni LI, O’Day BL, Killeen M, Harker H. 
Communicating about health care: observations from persons who are deaf or hard of hearing. Annals of Internal Medicine. 2004;140(5):356-62.

6. Maldonado MT, Canella P. Recursos de relacionamento para profissionais de saúde: a boa comunicação com clientes e seus familiares em consultórios, ambulatórios e hospitais. Rio de Janeiro: Editores Ltda: 2003.

7. Chaveiro N. Encontro do paciente surdo que usa língua de sinais com os profissionais da saúde [dissertação]. Goiânia (GO): Universidade Federal de Goiás; 2007.

8. Barbosa MA, Oliveira MA, Siqueira KM, Damas KCA, Prado MA. Linguagem brasileira de sinais: um desafio para a assistência de enfermagem. Rev Enferm UERJ. 2003;11(3):247-51.

9. Chaveiro N, Barbosa MA, Porto CC. Revisão de literatura sobre o atendimento ao paciente surdo pelos profissionais da saúde. Rev Esc Enferm USP. 2008;42(3):578-83.

10. Milbrath VM, Soares DC, Cecagno D, Amestoy SC, Siqueira HCH. Inserção social da criança portadora de necessidades especiais: a perspectiva materna. Cogitare Enferm. 2009;14(2):311-7.

11. Minayo MCS. O desafio do conhecimento: pesquisa qualitativa em saúde. São Paulo / Rio de Janeiro: Hucitec - Abrasco; 1992.

12. Bardin L. Análise de conteúdo. Lisboa: Edições 70; 1977.

13. Pagliuca LMF, Fiúza NLG, Rebouças CBA. Aspectos da comunicação da enfermeira com o deficiente auditivo. Rev Esc Enferm USP. 2007;43(3):411-8.

14. Porto CC, Teixeira CMFS. Linguagem dos órgãos e comunicação médico-paciente. JBM. J Bras Med. 2005;89(1):21-3.

15. Diniz D. Autonomia reprodutiva: um estudo de caso sobre a surdez. Cad Saúde Pública. 2003;19(1):175-81.

16. McAleer M. Communicating effectively with deaf patients. Nurs Stand. 2006;20(19)51-4.

17. Chaveiro N, Barbosa MA. Assistência ao surdo na área de saúde como fator de inclusão social. Rev Esc Enferm USP. 2005;39(4):417-22.

18. Barnett S. Communication with deaf and hard-of- hearing people: a guide for medical education. Acad Med. 2002;77(7):694-700. 